\title{
Should our approach to diuretic using in patients with gout change?
}

\author{
Duygu Tecer ${ }^{1 *}$ (D) Gozde Kubra Yardımc ${ }^{2}$, Alper Sari ${ }^{2}$ and Hakan Babaoglu ${ }^{3}$
}

We read with interest the recent article by Ranieri et al. [1]. The intention of the authors was to investigate the impact of diuretic therapy on the response of urate-lowering drugs (ULDs) in patients with gout. According to the results of this study, diuretic therapy did not impair response to ULDs. Also, there was no significant effect of diuretic use on the achievement of serum urate (SU) targets or on the maximum doses of ULDs. However, it is known that the European League Against Rheumatism (EULAR) has reported its concern in regard to the use of diuretics in patients with gout and recommends substitution of diuretic to losartan or calcium channel blockers if gout is diagnosed in a hypertensive patient receiving loop or thiazide diuretics [2]. For this reason, we believe that the results of this study, which reported the incompatibility with the recommendations of known and accepted organizations, should be interpreted carefully as it may bring a question mark to daily clinical practice.

First, the retrospective design of the study led to the heterogeneity of the patient population. Given the baseline characteristics of enrolled patients, 137 (65.9\%) patients in the allopurinol group and $30(85.7 \%)$ in the febuxostat group had hypertension. In addition, 107 (51.4\%) patients in the allopurinol group and 20 (57.1\%) patients in the febuxostat group had dyslipidemia. But in this study, we did not find any information about the drugs used for hypertension and dyslipidemia which may have an effect on urate metabolism. A large epidemiological study reported that calcium channel blockers and losartan are associated with a lower risk of incident gout among patients with hypertension [3]. It is well known that fenofibrate and statins have uricosuric properties $[4,5]$.

Second, in the study, alcohol use and dietary habits of patients may directly affect the SU levels. But there is no detailed information on alcohol use and dietary habits of patients. All of these factors may have affected the results of the study.

Lastly, given diuretic indications, $63 \%$ of patients using diuretics had pure hypertension without heart failure or chronic kidney disease. In these patients, although there are alternative anti-hypertensive drugs, the reason for the continuation of diuretics after diagnosis of gout is a matter to be discussed.

In conclusion, the results of this study, which can change our clinical practice, consisting of a retrospective and heterogeneous population, should be interpreted with caution. We believe that, as the authors emphasize, there is a need for prospective well-designed studies about the effects of diuretics on the necessary doses of ULDs to achieve the targeted SU level.

\footnotetext{
Authors' response

Comment on: Should our approach to diuretic use in patients with gout be changed?

Laura Ranieri ${ }^{1}$ Mariano Andres ${ }^{1,2}$

${ }^{1}$ Rheumatology Department, Hospital General Universitario de Alicante-ISABIAL, Alicante, Spain

Universidad Miguel Hernandez de Elche, Alicante, Spain
}

We thank Tecer and colleagues [6] for their interest and considerations addressed around our article [1]. They discuss whether the approach in the diuretic treatment in patients with gout should be changed according to our results

\footnotetext{
* Correspondence: duygu-tecer@hotmail.com

${ }^{1}$ Rheumatology Department, Mehmet Akif Inan Education and Research

Hospital, Ertugrul Street, Sanlıurfa 63300, Turkey

Full list of author information is available at the end of the article
}

and whether dietary habits or other agents used for hypertension or dyslipidemia might have acted as confounders.

The EULAR recommendations, in both the 2006 and 2016 versions, consider substituting diuretic therapy (loop agents or thiazides) when possible for the management of patients with gout [2, 7]. However, this recommendation was actually based on experts' opinion (graded as category of evidence 3, grade of recommendation $\mathrm{C}$ ) and was not 
unanimous (agreement of $8.2 \pm 0.9$ out of 10 in 2016). Our findings suggest that this approach may be unnecessary, at least to achieve the proper SU target to ensure crystal dissolution. SU was reduced below $6 \mathrm{mg} /$ $\mathrm{dL}$ in the majority of patients $(68.1 \%$ to $79.1 \%$ in the allopurinol group and $78.6 \%$ to $81.0 \%$ in the febuxostat group) and there were no differences according to diuretics. The rate of patients achieving stricter SU targets was also considerable. Along with this, the dosage of the ULDs was comparable between groups. We agree with the authors that these findings should be replicated in prospective controlled studies, but, in view of our results and recent reports [8], diuretics could be maintained. Certainly, the indication for diuretics may drive the decision. Whether diuretic withdrawal has an impact on future ULD maintenance dosing, in order to prevent the formation of new urate crystals, remains to be explored.

Alcohol and dietary intake were not registered in clinical records, but lifestyle advice is routinely given to all patients with gout and not led by whether they were on diuretics or not. The use of other agents with uricosuric action, such as calcium channel blockers, losartan, atorvastatin, or fenofibrate, was not registered. In our experience, their urate-lowering effect is modest, especially when they are combined with ULDs [9]. Following this, we do not routinely use them when we initiate ULDs. Thus, their impact on our study findings appears to be limited.

\section{Abbreviations \\ EULAR: European League Against Rheumatism; SU: Serum urate; ULD: Urate- lowering drug}

\section{Availability of data and materials}

Data sharing is not applicable to this article as no datasets were generated or analyzed during the current study.

\section{Authors' contributions \\ All named authors have contributed directly to the work with substantial contributions to conception and design and to drafting and revising the article critically for important intellectual content and final approval of the version to be published.}

\section{Ethics approval and consent to participate}

Not applicable. Our letter does not report on or involve the use of animal or human data or tissue.

\section{Consent for publication}

Not applicable.

\section{Competing interests}

The authors declare that they have no competing interests.

\section{Publisher's Note}

Springer Nature remains neutral with regard to jurisdictional claims in published maps and institutional affiliations.

\section{Author details}

${ }^{1}$ Rheumatology Department, Mehmet Akif Inan Education and Research Hospital, Ertugrul Street, Sanlıurfa 63300, Turkey. ${ }^{2}$ Department of Internal
Medicine, Division of Rheumatology, Hacettepe University, Faculty of Medicine, Ankara, Turkey. ${ }^{3}$ Department of Internal Medicine, Division of Rheumatology, Gazi University, Faculty of Medicine, Ankara, Turkey.

Published online: 26 October 2018

References

1. Ranieri L, Contero C, Peral ML, Calabuig I, Zapater P, Andres M. Impact of diuretics on the urate lowering therapy in patients with gout: analysis of an inception cohort. Arthritis Res Ther. 2018;20:53.

2. Richette P, Doherty M, Pascual E, Barskova V, Becce F, Castaneda-Sanabria J, et al. 2016 updated EULAR evidence-based recommendations for the management of gout. Ann Rheum Dis. 2017;76:29-42.

3. Choi HK, Soriano LC, Zhang Y, Rodriguez LA. Antihypertensive drugs and risk of incident gout among patients with hypertension: population based case-control study. BMJ. 2012;344:d8190.

4. Derosa G, Maffioli P, Sahebkar A. Plasma uric acid concentrations are reduced by fenofibrate: A systematic review and meta-analysis of randomized placebo-controlled trials. Pharmacol Res. 2015;102:63-70.

5. Ogata N, Fujimori S, Oka Y, Kaneko K. Effects of three strong statins (atorvastatin, pitavastatin, and rosuvastatin) on serum uric acid levels in dyslipidemic patients. Nucleosides Nucleotides Nucleic Acids. 2010;29:321-4

6. Tecer D, Yardimci GK, Sari A, Babaoglu H. Should our approach to diuretic using in patients with gout change? Arthritis Res Ther. 2018. https://doi.org/ 10.1186/s13075-018-1716-7

7. Zhang W, Doherty M, Bardin T, Pascual E, Barskova V, Conaghan P, et al. EULAR evidence based recommendations for gout. Part II: Management. Report of a task force of the EULAR Standing Committee for International Clinical Studies Including Therapeutics (ESCISIT). Ann Rheum Dis. 2006;65: 1312-24.

8. Kannangara DRW, Graham GG, Wright DFB, Stocker SL, Portek I, Pile KD, et al. Individualising the dose of allopurinol in patients with gout. Br J Clin Pharmacol. 2017:83:2015-26.

9. Waldman B, Ansquer JC, Sullivan DR, Jenkins AJ, McGill N, Buizen L, et al. Effect of fenofibrate on uric acid and gout in type 2 diabetes: a post-hoc analysis of the randomised, controlled FIELD study. Lancet Diabetes Endocrinol. 2018;6:310-8. 